\title{
Intercostal misplacement of a thoracic epidural catheter -A case report-
}

\author{
Young Bae Kim, and So Young Lee \\ Department of Anesthesiology and Pain Medicine, Korea Institute of Radiological and Medical Sciences, Seoul, Korea
}

Epidural block is useful for chronic pain and thoracic surgery. However, there are several complications, which include vessels or dural puncture, pleural puncture, misplacement of epidural catheter and trauma to the spinal cord. We report a case of intercostal misplacement of an epidural catheter in a 66-year-old patient who scheduled for left thoracotomy. (Korean J Anesthesiol 2010; 59: 344-347)

Key Words: Complication, Epidural catheter, Intercostal, Misplacement.

Epidural blocks for anesthesia are effective for post-operative pain management and chronic pain management, and especially after thoracic surgeries, which are followed by severe pain. Epidural blocks deter the reduction of the pulmonary function and they make the patient's respiratory management easier. However, the loss of resistance technique, which is most often used for identifying an epidural space, is a blind procedure. So vascular perforation, epidural puncture and pleural puncture can occur. Further, the final placement of the catheter may not be where it is supposed to be. This leads to not achieving the desired anesthetic effect. Inadvertent temporary or permanent damage to nerve tissue can also occur.

We inserted an epidural catheter for pain management in a patient who was undergoing a left upper lobectomy. Yet during the surgery, the thoracic surgeon discovered that the epidural catheter had been placed in the left intercostal space. We have examined our experience and here we present the case findings.

\section{Case Report}

A 66-year-old female American Society of Anesthesiologists physical status class III patient (weight: $62 \mathrm{~kg}$, height: $153 \mathrm{~cm}$ ) with pulmonary metastasis from thyroid cancer was to undergo a left thoracotomy. An epidural catheter was inserted into her for post-operative pain management. The patient's past medical history showed that she had undergone total thyroidectomy for thyroid cancer and she had been taking thyroid hormones. The pre-operative thyroid function test results showed that the T4 level was elevated to $14.27 \mathrm{ng} / \mathrm{ml}$. However, her blood tests, biochemical tests, urine tests and electrocardiography (EKG) were normal.

The patient was premedicated with glycopyrrolate $0.2 \mathrm{mg}$

Received: October 23, 2009. Revised: November 16, 2009. Accepted: December 15, 2009.

Corresponding author: So Young Lee, M.D., Department of Anesthesiology and Pain Medicine, Korea Institute of Radiological and Medical Sciences, 215-4, Gongneung-dong, Nowon-gu, Seoul 139-240, Korea. Tel: 82-2-970-2600, Fax: 82-2-970-2161, E-mail: soyo6761@hanmail.net (c) This is an open-access article distributed under the terms of the Creative Commons Attribution Non-Commercial License (http:// creativecommons.org/licenses/by-nc/3.0/), which permits unrestricted non-commercial use, distribution, and reproduction in any medium, provided the original work is properly cited. 
and midazolam $2.0 \mathrm{mg}$ intramuscularly one hour before arrival in the operating room. The EKG, noninvasive blood pressure manometer and pulse oximeter were attached when the patient was on the operating table. One hundred \% oxygen was administered for anesthetic induction. Thiopental sodium $300 \mathrm{mg}$ was intravenously infused and loss of consciousness was observed. Vecuronium bromide $7 \mathrm{mg}$ and fentanyl $25 \mu \mathrm{g}$ were administered. A 35 Fr. double lumen endotracheal tube was used for the tracheal intubation via direct laryngoscopy. The exact location of the endotracheal tube was found by performing fiberoptic bronchoscopy. Anesthesia was maintained by managing the oxygen at $2 \mathrm{~L} / \mathrm{min}$, the nitrogen oxide at $2 \mathrm{~L} / \mathrm{min}$ and the sevoflurane at the $1-2.0$ vol\% range. Vecuronium bromide was additionally administered when necessary for muscle relaxation.

After arterial cannulation in the left radial artery, a central venous catheter was inserted in the right subclavian vein. To perform the patient-controlled epidural analgesia, the patent was moved from the supine position to the right lateral position combined with the knee-chest position. The median approach using an $18 \mathrm{G}$ Tuohy needle was attempted in the space between the spinous processes of T-7 to T-8, but the approach could not be done, so it was switched to the paramedian approach. The needle was advanced. The loss of resistance technique with air was used to identify the epidural space. The loss of resistance was noted $7.2 \mathrm{~cm}$ beneath the skin surface. Next the Tuohy needle's bevel was placed towards the cephalad direction. The epidural catheter was then advanced 3 more centimeters. Loss of resistance was not felt during the advancement. We checked that the needle aspiration did not contain any CSF or blood. The catheter was then secured. As a test, $2 \%$ lidocaine $60 \mathrm{mg}$ was injected in the catheter, but there was no change in the vital signs. Afterwards, the location of the tubes was checked with

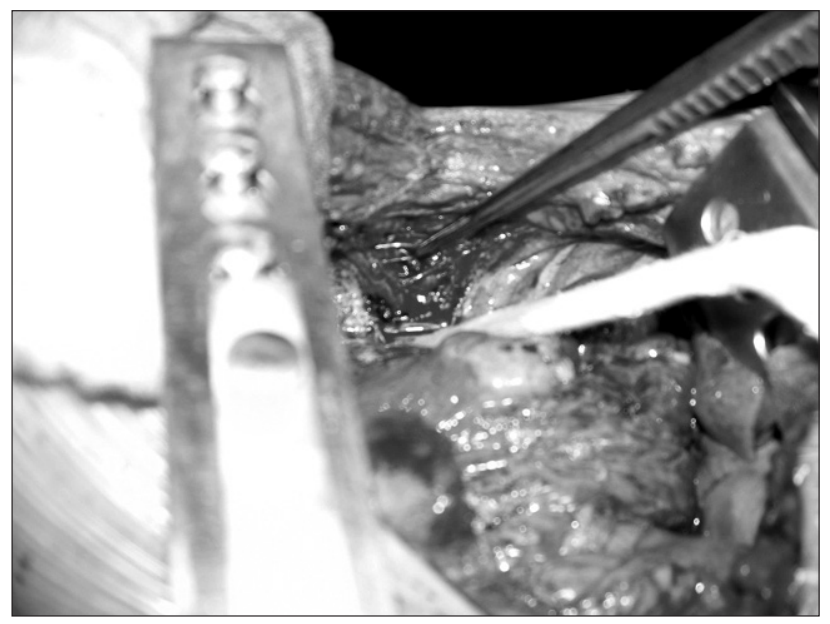

Fig. 1. Thoracic epidural catheter lying in the intercostal space. fiberoptic bronchoscopy.

The surgery proceeded without any complications. Three hours into the surgery, the thoracic surgeon discovered the epidural catheter in the left intercostal space (Fig. 1 and 2). We did not find hemorrhaging or tissue damage caused by the catheter. The catheter was immediately removed. The surgery ended with no other problems. The pain management was replaced with patient-controlled analgesia.

After the surgery, the endotracheal tube was extubated, and the patient was moved to the intensive care unit. The postoperative pain management was satisfactory. There was no paresthesia or diskinesia in the lower extremities. The thoracic X-ray showed no abnormalities. The patient recovered with no apparent sequelae and he was discharged on the 12th postoperative day.

\section{Discussion}

The pain from a thoracotomy not only causes discomfort, but it also limits deep breathing and coughing. This can cause pulmonary complications, an elevated blood pressure and increased oxygen consumption by the heart, and all this can lead to cardiovascular complications [1]. A thoracotomy is not just a surgical procedure. After the surgery, a chest tube must be inserted, so thoracotomy is one of the most painful surgeries. The pain can last up to 2 months. In $30 \%$ of the patients, the pain becomes chronic [2]. Therefore, appropriate pain management is very important because it not only promotes comfort, but it helps prevent postoperative complications as well as reducing the morbidity and mortality [3].

Of the various methods of pain management, epidural blocks neither suppress sympathetic nerves nor obstruct motor or sensory nerves. They allow for easy pain management. Epidural blocks in general are known to have a longer term

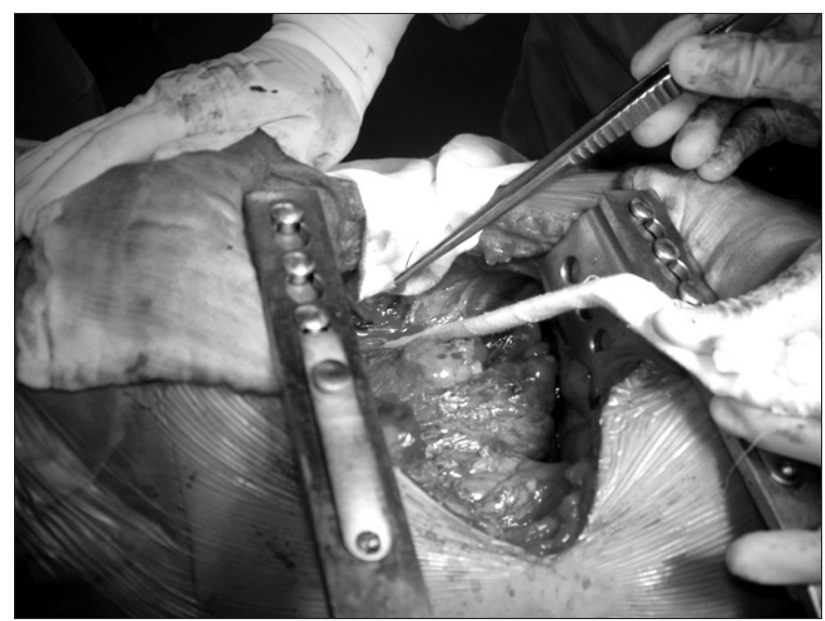

Fig. 2. Using forceps to identify epidural catheter more clearly. 
of pain alleviation and they more effectively manage pain than intravenous analgesia. So they are often used in patients who undergo thoracic and epigastric surgeries. However, the drawback is that the procedure is difficult with the side-effects ranging from minor to lethal. Such side-effects occur in $2-8 \%$ of the patients who undergo epidural blocks, which is quite often, and even when the epidural blocks are performed by very skilled anesthesiologists [4]. The misplacement of catheters can happen. The most common area they are misplaced is in the intervertebral foramen. Failed pain management or spinal/ tissue damage may occur when the catheter is misplaced [5].

In the cases of combined general anesthesia and epidural anesthesia, there are many arguments on the appropriate timing for placement of the epidural catheter. Some consider that the indicative pain or signs following a misplaced catheter may make it better to insert the epidural catheter when the patient is conscious, But conscious patients have also been reported to have experienced direct spinal damage during performance of spinal anesthesia [6], and catheters have entered the spinal cord without causing pain during the performance of epidural blocks [7]. In Korea, an epidural catheter that was inserted into a conscious patient was suddenly was located in the thorax without any signs [8]. So catheter insertion while the patient is conscious may just simply increase his/her discomfort.

The paramedian approach has been reported be a less anatomically dangerous epidural technique than the median approach. But it is more difficult to check the location of the epidural space with the loss of resistance technique using the paramedian approach than it is using the median approach, so side-effects occur more frequently with using the paramedian approach. However, such side-effects may occur even when using the median approach. To reduce the side-effects, softer catheters may reduce perforations. The insertion site of the Tuohy needle should be kept as close as possible to the final destination of the epidural catheter [9].

In the present study, the loss of resistance site that was mistaken for the epidural space was the left intercostal space. The catheter inserted afterwards was coiled $3 \mathrm{~cm}$ in the intercostal space. It was discovered during the process of hemostasis before the end the surgery. There is a method for blocking the intercostal nerves. The catheter remains in the intercostal space or the thoracic cavity, infuse the local anesthesia and let it diffuse through the parietal pleura and intercostal muscle and let it reach the intercostal nerves. However, this method makes it likely that that the catheter and the peripheral tissue might be sutured together during wound closure [10]. In the present case, it was not clear whether there was peripheral tissue damage during the catheter insertion.

The post-operative loss of local anesthesia may have occurred through the thoracic duct, and the pain-alleviating effects could not be verified. So the catheter was removed and replaced with intravenous patient-controlled analgesia.

Many previously reported cases have had the catheter placed in the thoracic cavity ipsilateral to the surgery site $[4,8,9,11]$. There have also been reports of unilateral pleural punctures $[12,13]$. Although this is the first such report from Korea, there was a similar case overseas where the thoracic epidural catheter was placed in the intercostal space ipsilateral to the surgery site [14].

In conclusion, misplacement of the epidural catheter can happen when using both the paramedian and median approaches and this can occur both ipsilateral and unilateral to the surgical area. Therefore, when the epidural block does not have enough analgesic effect or the effect is only on the unilateral side, the location of the catheter should be checked to see if it is correct. There is currently no known method for decreasing the side-effects of epidural blocks. Moreover, there is much controversy on the issue of whether the patient should be conscious during epidural blocks. Other countries have generally started to use imaging devices, such as ultrasound and C-arm fluoroscopy, when performing epidural blocks. This compensates for not being able to see the location of the Tuohy needle and the catheter with the naked eye and it reduces the likelihood of side-effects. It may also help to more quickly find misplaced catheters.

\section{References}

1. Conacher ID. Pain relief after thoracotomy. Br J Anaesth 1990; 65: 806-12.

2. De Cosmo G, Aceto P, Gualtieri E, Congedo E. Analgesia in thoracic surgery: review. Minerva Anestesiol 2009; 75: 393-400.

3. Torda TA, Pybus DA. Clinical experience with epidural morphine. Anaesth Intensive Care 1981; 9: 129-34.

4. Zaugg M, Stoehr S, Weder W, Zollinger A. Accidental pleural puncture by a thoracic epidural catheter. Anaesthesia 1998; 53: 69-71.

5. Halpenny DG, Loken RG, Williams JA. Transforaminal escape of a lumbar epidural catheter. Can J Anaesth 1992; 39: 594-5.

6. Absalom AR, Martinelli G, Scott NB. Spinal cord injury caused by direct damage by local anaesthetic infiltration needle. Br J Anaesth 2001; 87: 512-5.

7. Katz N, Hurley R. Epidural anesthesia complicated by fluid collection within the spinal cord. Anesth Analg 1993; 77: 1064-5.

8. Kim JY, Park JS, Jeong CI, Lee SI, Kim KT, Choe WJ, et al. Accidental intrapleural positioning of an epidural catheter in a patient undergoing a right pneumonectomy: a case report. Korean J Anesthesiol 2008; 55: 494-7.

9. Furuya A, Matsukawa T, Ozaki M, Kumazawa T. Interpleural misplacement of an epidural catheter. J Clin Anesth 1998; 10: 425-6.

10. Kim SH, Yu BS, Lim KJ, So KY, An TH, Chung CD. A comparison of the effect between intravenous fentanyl and interpleural bupivacaine following thoracoscopic surgery. Korean J Anesthesiol 2002; 42: 620-6. 
11. Eti Z, Lacin T, Yildizeli B, Dogan V, Gögüs FY, Yüksel M. An uncommon complication of thoracic epidural anesthesia: pleural puncture. Anesth Analg 2005; 100: 1540-1.

12. Iida Y, Kashimoto S, Matsukawa T, Kumazawa T. A hemothorax after thoracic epidural anesthesia. J Clin Anesth 1994; 6: 505-7.
13. Shime N, Shigemi K, Hosokawa T, Miyazaki M. Intrathoracic migration of an epidural catheter. J Anesth 1991; 5: 100-2.

14. Tsui BC, Kulkarni P. Migration of a thoracic epidural catheter into the intercostal space via the intervertebral foramen. Anesth Analg 2009; 108: 371-3. 